
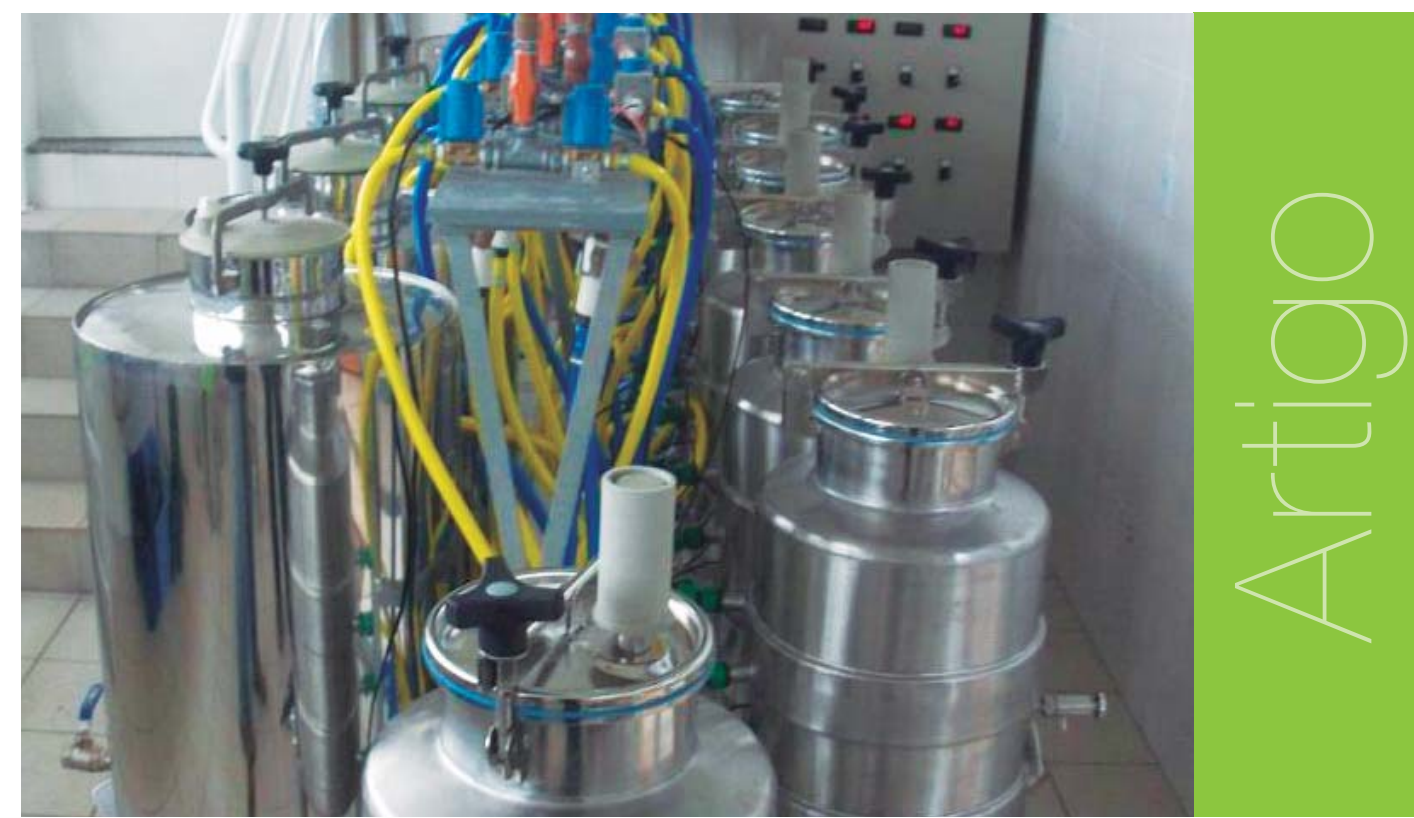

\title{
Ações do Laboratório de Viticultura e Enologia da Udesc Lages no desenvolvimento da vitivinicultura de altitude de Santa Catarina
}

\author{
Douglas André Wurz - douglaswurz@hotmail.com \\ José Luiz Marcon Filho² - marconfilho_j|@yahoo.com.br \\ Ricardo Allebrandt ${ }^{3}$ - ricardoudesc@gmail.com \\ Leo Rufato4 - leoruffato@yahoo.com.br
}

\section{RESUMO}

0 objetivo desse trabalho foi descrever as atividades relacionadas à extensão universitária realizada pelo Laboratório de Enologia da Universidade do Estado de Santa Catarina. Verificase uma crescente no número de projetos de graduação e pós-graduação. Em quatro anos de atividades, foram onze artigos científicos publicados em periódicos, onze trabalhos completos, dez resumos expandidos, trinta e sete resumos simples publicados em anais de eventos científicos, vinte textos em jornais e dez cursos de capacitação realizados por acadêmicos envolvidos no Laboratório. Desde a safra 2013, foram vinificadas $11.308 \mathrm{~kg}$ de uvas, sendo 52\% desse total destinados à pesquisa científica e 48\% destinados à extensão rural, atendendo nesse período 16 produtores rurais da região do planalto catarinense.

\footnotetext{
1 Engenheiro Agrônomo, doutorando em Produção Vegetal CAV/UDESC

2 Engenheiro Agrônomo, doutor em Produção Vegetal CAV/UDESC

3 Engenheiro Agrônomo, doutorando em Produção Vegetal CAV/UDESC

4 Engenheiro Agrônomo, Doutor Professor Fruticultura CAV/UDESC
} 


\section{PALAVRAS-CHAVE}

Extensão universitária. Vinhos de altitude. Conhecimento técnico-científico. Desenvolvimento regional.

\section{ABSTRACT}

The objective of this work was to describe the activities related to the university extension carried out by the Oenology Laboratory of the State University of Santa Catarina. There is a growing number of undergraduate and graduate projects. In four years of activities, there were eleven scientific articles published in journals, eleven complete papers, ten expanded abstracts, thirty-seven simple abstracts published in annals of scientific events, twenty texts in newspapers and ten training courses by the academics involved in the Laboratory. Since the harvest of 2013, 11,308 $\mathrm{kg}$ of grapes have been vinified, $52 \%$ of this total being destined to scientific research and $48 \%$ destined to rural extension, serving in that period 16 rural producers of the region of the Santa Catarina plateau.

\section{KEYWORDS}

University extension. Wines of altitude. Technical-scientific knowledge. Regional development.

\section{Introdução}

A vitivinicultura tem sido uma atividade importante para a economia de algumas regiões, principalmente, daquelas localizadas no Sul do país, onde se concentra o maior volume de produção de uva, vinho e demais derivados. A importância do setor para essas regiões está inteiramente relacionada à sustentabilidade das pequenas e médias empresas rurais, que produzem uva de mesa e uva para processamento bem como daquelas que atuam no sistema de vinificação, no sentido de contribuir para a geração de emprego e renda para a economia (DUARTE, 2013).

Até 0 final da década de 90, o Rio Grande do Sul deteve praticamente exclusividade na produção, elaboração e comercialização de vinhos finos no Brasil, representando 95\% da produção nacional (PROTAS et al., 2002). No entanto, a partir da década 90, houve um marco referencial da política do setor empresarial, no sentido de promover melhorias na estrutura produtiva da vitivinicultura brasileira, quando começaram a ocorrer investimentos, tanto na implantação e/ou modernização das vinícolas localizadas nas regiões tradicionais quanto nos novos polos produtores (PROTAS, 2008).

Com essas mudanças, verificou-se a emergência de novas regiões produtoras com safras cada vez maiores, produtividade acima da média mundial e produção de uvas de mesa e vinhos finos de alta qualidade. Nesse contexto, verificou-se a vitivinicultura nacional se transformando e crescendo qualitativamente nos últimos anos, com destaque para novos polos produtores em regiões não tradicionais. Destaca-se como polo emergente da viticultura brasileira, as regiões de altitude do planalto catarinense, como o município de São Joaquim. Situada a $28^{\circ} \mathrm{S}$ e $49^{\circ} \mathrm{W}$, com altitude entre $950 \mathrm{~m}$ e $1.400 \mathrm{~m}$, esse polo produtor está voltado principalmente ao cultivo de castas de Vitis vinifera L. para a produção de vinhos finos.

A vitivinicultura nas regiões de altitude do estado Santa Catarina, embora recente, apresenta grande importância econômica, sendo o estado, o segundo maior produtor de uvas destinadas a elaboração de vinhos finos no Brasil. Observa-se nessas regiões ciclos fenológicos mais prolongados em comparação a outras regiões vitivinícolas brasileiras, isso ocorre pelas menores temperaturas noturnas e maior amplitude térmica, produzindo uvas com elevada qualidade enológica (MALINOVSKI et al., 2016).

No entanto, pelo fato desta região não ser tradicionalmente produtora de uvas, ainda são necessários ajustes de técnicas de manejo e de elaboração de vinhos finos específicos. Além disso, por ser um novo polo vitícola, há pequenos produtores que não possuem infraestrutura adequada para a elaboração de vinhos de qualidade.

Baseado no descrito acima e na problemática enfrentada pelos produtores da região de altitude do planalto sul de Santa Catarina, a Universidade do Estado de Santa Catarina (Udesc), localizada no município de Lages-SC, vem desenvolvendo pesquisas para solucionar 
problemas referentes ao cultivo da videira nessa região. Além de atuar no desenvolvimento de pesquisas na área agronômica, a Udesc Lages atua na prestação de serviços aos viticultores da região, realizando vinificações em pequena escala para os produtores de uva que não possuem estrutura para elaborar o vinho e capacitando os técnicos e vitivinicultores na aplicação de técnicas de produção mais eficientes.

Nesse contexto, a Udesc Lages inaugurou, em 05/04/2012, o Laboratório de Enologia (Figura 1), em parceria com o governo do Estado de Santa Catarina e o Fundo de Amparo à Pesquisa e Inovação do Estado de Santa Catarina (Fapesc). 0 Laboratório de Enologia tem a finalidade de vinificar, em pequena escala, uvas provenientes das regiões de altitude de Santa Catarina e, para tanto, possui equipamentos para cada etapa da elaboração de vinhos em escala semiindustrial, permitindo a produção de vinhos de qualidade (Figura 2).

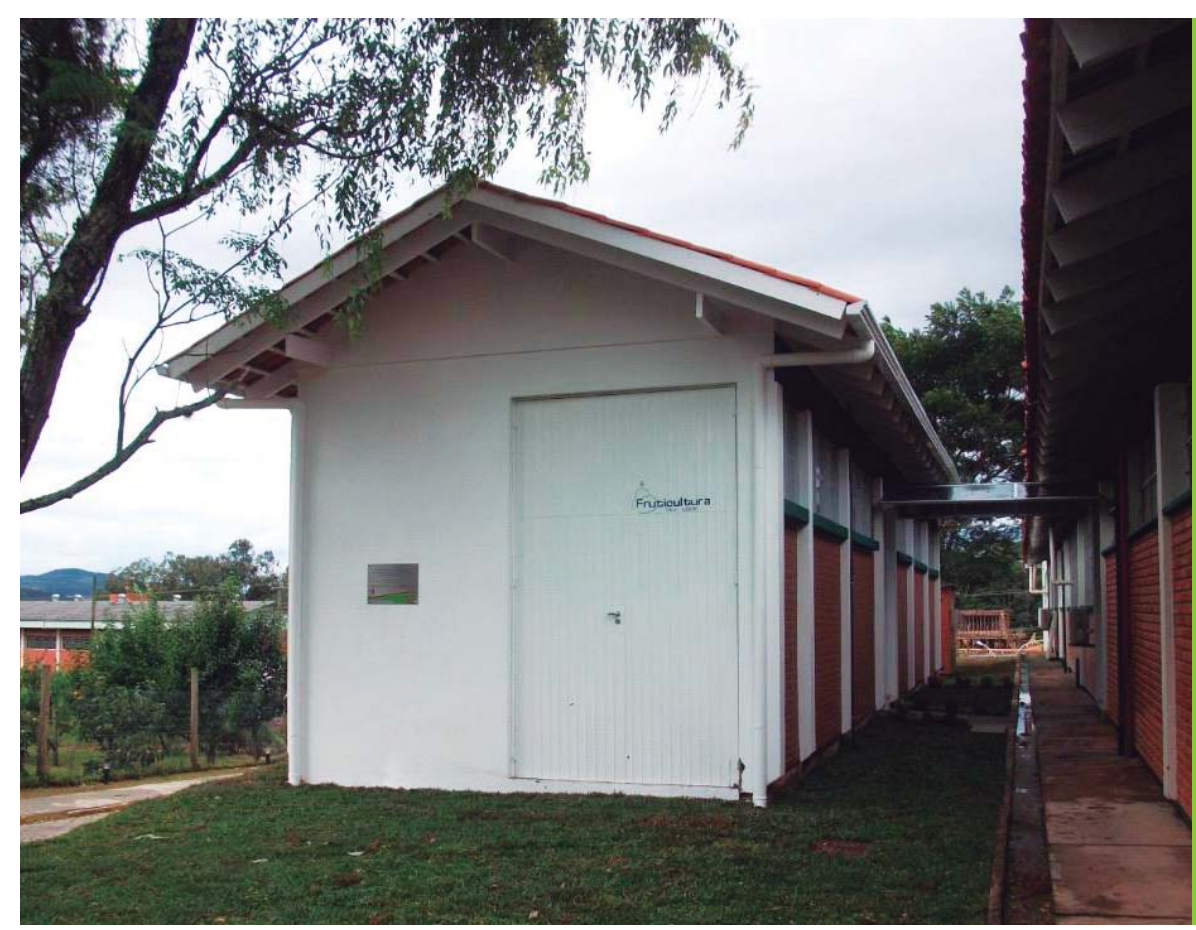

Figura 1: Laboratório de

Enologia da Universidade do Estado de Santa Catarina, Lages-SC.

Foto: José Luiz Marcon Filho

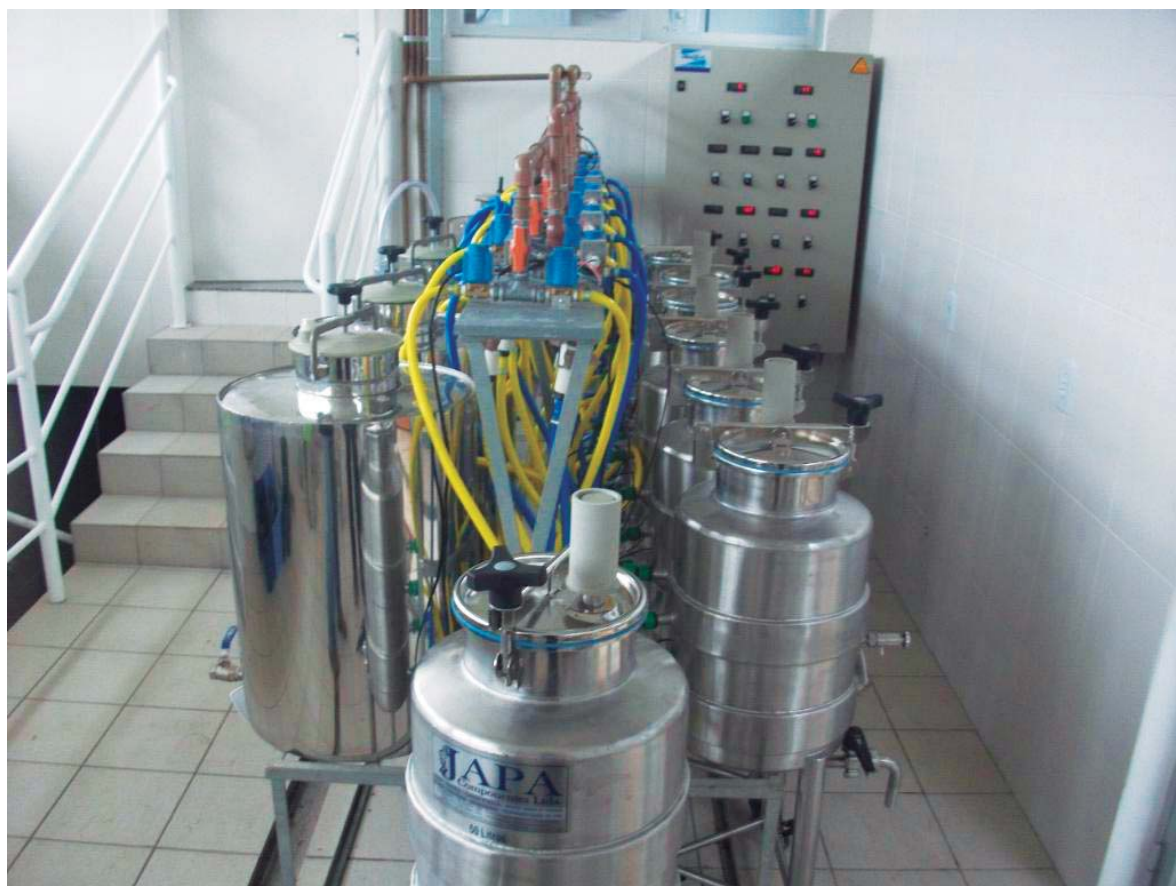

Figura 2: Tanques

de fermentação com

controle automatizado de temperatura.

Foto: José Luiz Marcon Filho 
0 objetivo desse trabalho é descrever as atividades de extensão universitária realizadas pelo Laboratório de Enologia do câmpus Lages da Udesc bem como fazer um levantamento descritivo das atividades realizadas no âmbito da pesquisa e ensino, articulando a relação universidade e sociedade através da extensão universitária.

\section{Metodologia}

0 trabalho foi desenvolvido na Universidade do Estado de Santa Catarina, câmpus Lages - SC. As atividades descritas no trabalho seguem a premissa de possuir um cunho científico em conjunto com ações de extensão, que visam a divulgação dos resultados obtidos através das pesquisas realizadas pela instituição bem como a prestação de serviços a produtores de uva na região de altitude do planalto sul de Santa Catarina.

Essa pesquisa classifica-se como descritiva, que segundo Cooper e Schindler (2011), visa à descriçãa de fatos ou atributos da amostra da pesquisa. Nesse sentido, foi realizado um levantamento de dados sobre a atuação do Laboratório de Enologia da Udesc Lages nas esferas ensino, pesquisa e extensão relacionadas à área de viticultura e enologia.

Os dados coletados foram: o número de projetos de pesquisa de iniciação científica, mestrado, doutorado e pós-doutorado realizados no Laboratório; o número de publicações científicas e informativas referentes ao tema vitivinicultura; as quantidades $(\mathrm{kg})$ de uva processada provindas de projetos de pesquisa e de produtores; número de produtores atendidos.

Os números referentes aos de projetos de pesquisa foram obtidos com os professores orientadores e alunos envolvidos nos mesmos. 0 número de publicações científicas e informativas foi obtido através de pesquisas em plataformas de pesquisa de periódicos científicos, além de jornais e anais de congressos e outros eventos das áreas de viticultura, enologia e fruticultura. As quantidades de uvas processadas e o número de produtores atendidos foram obtidas nos históricos de vinificação do Laboratório. Além desses dados, foram contabilizados os cursos e treinamentos ministrados pelos responsáveis do Laboratório de Enologia, com o objetivo de divulgar as informações obtidas em pesquisas e capacitar técnicos e estudantes sob aspectos relacionados à produção da uva, elaboração e degustação de vinhos.

Finalizada a coleta de dados, foram gerados tabelas e gráficos informativos para a apresentação e discussão dos resultados. A análise e interpretação desses foram realizadas de forma ampla, correlacionando com outros conhecimentos e comparando a outros estudos do gênero.

\section{Resultados e discussão}

As atividades extensionistas surgem da necessidade de uma interação entre universidade e sociedade e são de suma importância por serem fonte de aprendizagem e de "oxigenação" do conhecimento (artístico, científico, tecnológico e cultural) produzido na universidade, possibilitando a geração de novos conhecimentos de forma interdisciplinar através de suas ações (SANTOS, 2012), tendo como objetivo a promoção da ligação entre a universidade e a comunidade local, representando o compartilhamento de conhecimentos e saberes (TERRA; ZÜGE, 2013).

A extensão universitária na Lei de Diretrizes e Bases da Educação Nacional no 9.394 de 1996 é considerada um processo educativo, cultural e científico que articula 0 ensino e a pesquisa de forma indissociável e viabiliza a relação transformadora entre universidade e sociedade (SUGAHARA, 2012).

Nas regiões de altitude, devido às características particulares e ao cultivo recente de uvas viníferas, há um déficit de informações técnicas que contribuem para 0 direcionamento adequado dos vinhedos a fim de se obter vinhos de qualidade. A maioria das técnicas de manejo empregadas nos vinhedos foi baseada nas experiências de produtores e técnicos bem como nos resultados de pesquisas oriundas de outras regiões vitivinícolas. Nesse sentido, o Laboratório de Enologia da Universidade do Estado de Santa Catarina, através dos seus programas de 
pós-graduação e graduação, vem trabalhando junto aos viticultores da região com o objetivo de desenvolver informações técnicas que estejam adequadas à realidade da região.

Isso reflete no número de projetos de pesquisas que vêm sendo desenvolvidos vinculados ao Laboratório de Enologia, conforme destacado na Tabela 1, onde observa-se que desde 2013 foram realizados ou estão em andamento quatro projetos de iniciação científica vinculados à graduação do Centro de Ciências Agroveterinárias da Universidade do Estado de Santa Catarina, nove dissertações de mestrado, seis teses de doutorado e um projeto de pós-doutorado vinculados aos programas de pós-graduação de Produção Vegetal da Universidade do Estado de Santa Catarina, gerando informações técnicas quanto a uma série de temas, tais como: sistemas de condução, manejo fitossanitário, porta-enxertos, manejo do dossel vegetativo e potencial vitícola de novas variedades para a elaboração de vinhos finos e espumantes (Tabela 1).

\begin{tabular}{lccc} 
Nivel & Concluido & Andamento & Total \\
\hline Iniciação científica & 2 & 2 & 4 \\
\hline Dissertação & 6 & 3 & 9 \\
\hline Tese & 3 & 3 & 6 \\
\hline Pós-doutorado & 1 & 0 & 1 \\
\hline
\end{tabular}

Tabela 1: Número de projetos de iniciação cientifica, dissertacões de mestrado, teses de doutorado e pós-doutorado realizados no Laboratório de Enologia da Universidade do Estado de Santa Catarina (Udesc), Lages/SC

Com os projetos de pesquisa que vêm sendo desenvolvidos, o Laboratório de Enologia, através dos programas de graduação e pós-graduação do Centro de Ciências Agroveterinárias, vem alcançando seus objetivos de gerar informações técnicas e de pesquisa e fazer com que elas cheguem até os viticultores e os técnicos que atuam junto aos vinhedos.

Em quatro anos, foram publicados onze artigos científicos em periódicos, onze trabalhos completos em anais de eventos científicos, dez resumos expandidos em anais de eventos científicos, trinta e sete resumos simples em anais de eventos científicos, vinte textos informativos em jornais e ministrados dez cursos de capacitação técnica (Tabela 2).

\begin{tabular}{lcccccc} 
& \multicolumn{5}{c}{ Ano } & Total \\
\cline { 2 - 5 } Publicação & $\mathbf{2 0 1 3}$ & $\mathbf{2 0 1 4}$ & $\mathbf{2 0 1 5}$ & $\mathbf{2 0 1 6}$ & \\
\cline { 2 - 5 } Artigos científicos & 0 & 0 & 4 & 7 & 11 \\
\hline Trabalhos completos & 0 & 4 & 2 & 5 & 11 \\
\hline Resumos Expandidos & 2 & 0 & 7 & 1 & 10 \\
\hline Resumos simples & 7 & 8 & 4 & 18 & 37 \\
\hline Textos em jornais & 0 & 0 & 8 & 12 & 20 \\
\hline Cursos ministrados & 1 & 1 & 3 & 5 & 10 \\
\hline
\end{tabular}

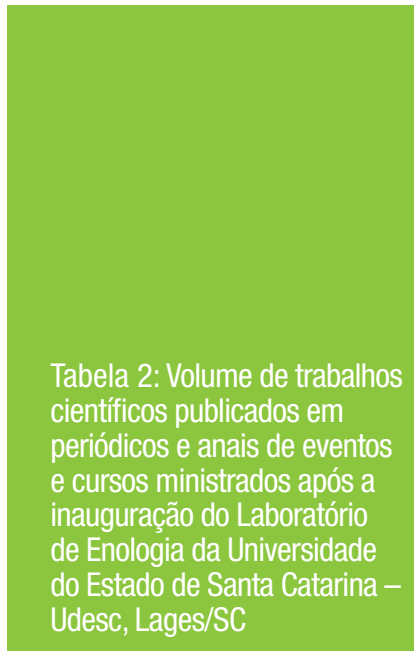

A partir do acompanhamento da vitivinicultura nas regiões de altitude de Santa Catarina já foram publicados trabalhos sobre porta-enxertos (ALLEBRANDT et al., 2015), manejo do dossel vegetativo (MACEDO et al., 2015), raleio de cachos (MARCON FILHO et. al., 2015), sistemas de condução e fitossanidade (DE BEM et al., 2015; DE BEM et al., 2016a), poda invernal (MARCON FILHO et al., 2016), enoturismo (WÜRZ et al., 2016), danos causados por geadas tardias (MARCON FILHO et al., 2016), fitorreguladores (KRETZSCHMAR et al., 2016; RUFATO et al., 2016) e variedades resistentes a doenças fúngicas (DE BEM et al., 2016b). 
Por possuir toda a estrutura de vinificação semi-industrial, os acadêmicos de pós-graduação que atuam no Laboratório de Enologia ministram cursos de elaboração de vinhos com 0 objetivo de capacitar produtores, técnicos e acadêmicos que tenham interesse na elaboração de vinhos em pequena escala. Além da elaboração dos vinhos, são ministrados cursos de degustação de vinhos e espumantes.

0 Brasil tem registrado crescimento no consumo de vinhos e espumantes, apesar do consumo per capita ainda ser muito baixo. Enquanto cada brasileiro consome em média cerca de 50 litros de cerveja e 6 de cachaça ao ano, o consumo de vinhos finos fica em torno de 2 litros per capita ao ano (COPELLO, 2015). Portanto, é fundamental capacitar os consumidores sobre as técnicas de degustação de vinhos, com o objetivo de aumentar o consumo per capita deste produto.

Além de gerar informações técnico-científicas, um dos grandes objetivos do Laboratório de Enologia é prestar serviço de extensão a pequenos produtores, prestando serviços de vinificação em pequena escala. Ao longo de quatro anos de atividades do Laboratório de Enologia, foram vinificados $11.308 \mathrm{~kg}$ de uvas, sendo 52\% destinadas à vinificação de experimentos científicos e 48\% destinados à vinificação de produtores (Tabela 3), atendendo nesse período 16 pequenos produtores rurais que não possuem estrutura de vinificação em suas propriedades.

Tabela 3: Quantidade total

(kg) de uvas vinificadas pelo Laboratório de Enologia da Universidade do Estado de Santa Catarina (Udesc), Lages/SC, e percentuais destinadas à pesquisa científica e extensão rural

\begin{tabular}{|c|c|c|c|c|c|}
\hline \multirow{2}{*}{ Microvinificação } & \multicolumn{4}{|c|}{ Safra } & \multirow{2}{*}{ Total } \\
\hline & 2013 & 2014 & 2015 & 2016 & \\
\hline Quantidade (kg) & 2.158 & 1.679 & 3.702 & 3.769 & 11.308 \\
\hline Pesquisa & $71 \%$ & $66 \%$ & $58 \%$ & $28 \%$ & $52 \%$ \\
\hline Extensão & $29 \%$ & $34 \%$ & $42 \%$ & $72 \%$ & $48 \%$ \\
\hline Produtores atendidos & 4 & 4 & 5 & 3 & 16 \\
\hline
\end{tabular}

Verifica-se um aumento ano a ano do volume de serviços destinados a pequenos produtores rurais. Em 2013, a vinificação destinada à extensão representava 29\%, já em 2016, esse percentual subiu para $72 \%$. Isso se deve a um aperfeiçoamento da metodologia de microvinificação dos experimentos científicos, adotando metodologia descrita por Marcon Filho (2016), onde reduziuse a necessidade de grandes quantidades de uva para as microvinificações experimentais, otimizando a utilização de equipamentos e, consequentemente, sendo possível vinificar uma maior quantidade de uvas $(\mathrm{kg})$ oriundas de pequenos produtores rurais.

Ressalta-se que a relação entre ensino, pesquisa e extensão reafirma a extensão como processo acadêmico, visto que pode trazer uma rica experiência acumulada: 0 deslocamento do eixo pedagógico clássico professor-aluno para 0 eixo aluno e comunidade, com a atuação do professor como coparticipante e orientador (CORRÊA, 2003). Além disso, para a complexa sociedade em que se vive, a extensão universitária configura-se em uma das formas de atuação mais necessárias, pois a universidade é uma realidade social e política, uma instituição educacional que expressa a sociedade da qual faz parte e com isso a extensão universitária visa a favorecer a capacitação dos acadêmicos para 0 agir profissional, colocando-os em contato direto com a realidade social (SANTOS, 2012).

Destaca-se também a importância do Laboratório de Enologia para aulas práticas dos cursos de Agronomia e da pós-graduação em Produção Vegetal, na qual os acadêmicos podem acompanhar todo o processo de elaboração de vinhos. 0 que faz com que 0 Laboratório de Enologia desempenhe suas funções de ensino, pesquisa e extensão. Nesse sentido, para se abordar satisfatoriamente a questão da extensão universitária brasileira, faz-se necessário não perder de vista suas articulações com as demais funções básicas da universidade: 0 ensino e a pesquisa (SANTOS, 2012). 


\section{Conclusões}

0 Laboratório de Enologia do Centro de Ciências Agroveterinárias da Universidade do Estado de Santa Catarina está em plena atividade desde 2013 e já é possível verificar sua contribuição para o desenvolvimento da viticultura nas regiões de altitude de Santa Catarina, gerando informações técnicas que contribuem para o desenvolvimento social dos envolvidos e consolidação da atividade nessas regiões.

Além disso, o Laboratório de Enologia desempenha um papel fundamental na prestação de serviços a pequenos produtores da região de altitude que não possuem estrutura para a elaboração de vinhos finos.

\section{Referências}

ALLEBRANDT, R.; MARCON FILHO, J.L.; BEM, B.P.; WÜRZ, D.A.; BRIGHENTI, A.F.; KRETZSCHMAR, A.A.; RUFATO, L. Fenologia da variedade merlot produzida sobre três portaenxertos em elevadas altitudes de Santa Catarina. Revista Brasileira de Viticultura e Enologia, n. 7, p. 36-43, 2015.

BEM, B. P.; BOGO, A.; EVERHART, S.; CASA, R.T.; GONÇALVES, M.J.; MARCON FILHO, J.L.; CUNHA, I. Effect of Y-trellis and vertical shoot positioning training systems on downy mildew and botrytis bunch rot of grape in highlands of southern Brazil. Scientia Horticulturae, v. 185, p. 162-166, 2015.

BEM, B.P.; BRIGHENTI, E.; BONIN, B.F.; ALLEBRANDT, R.; ARAÚJO, L.; BRIGHENTI, A.F.; BOGO, A. Downy mildew intensity in tolerant grapes varieties in highlands of Southern Brazil. BIO Web of Conference, v.7, p.1-4, 2016.

BEM, B.P.; BOGO, A.; EVERHART, S.; CASA, R.T.; GONÇALVES, M.J.; MARCON FILHO, J.L; RUFATO, L.; SILVA, F.N.; ALLEBRANDT, R.; CUNHA, I. Effect of four training systems on the temporal dynamics of downy mildew in two grapevine cultivars in southern Brazil, Tropical Plant Pathology, v.41, p.370-379, 2016.

COOPER, Donald R.; SCHINDLER, Pamela S. Métodos de pesquisa em administração. 10ª Edição, Porto Alegre: Bookman, 2011

COPELLO, M. Brasil, mercado em ebulição. Revista Vinhos do Brasil. Rio de Janeiro. p.34-37. 2015.

CORRÊA, E.J. Extensão universitária, política institucional e inclusão social. Revista Brasileira de extensão universitária, v.1, n.1, p.12-15, 2003.

DUARTE, V.N. Estudo da cadeia produtiva do vinho em Santa Catarina: características e estágio atual. Evidências, v.13 n.1 p.45-56, 2013.

KRETZSCHMAR, A.A., LERIN, S., FAGHERAZZI, A.F., MARIO, A.E., BASTOS, F.E.A., ALLEBRANDT, R. and RUFATO, L. Application of abscisic acid increases the colour of 'Rubi' grape berries in Southern Brazil. Acta Horticulturae, v.1115, p.231-236, 2016.

MACEDO, T. A.; MARCONFILHO, J.L.; BRIGHENTI, A.F.; SILVA, L.C.; RUFATO, L.; KRETZSCHMAR, A.A. Manejo do dossel vegetativo e qualidade físico-química dos cachos de 'sangiovese' e 'tempranillo' em região microclimática de altitude. Revista de Ciências Agroveterinárias, Lages, v. 14, n. 2, p. 146-152, 2015.

MALINOVSKI, L.I.; BRIGHENTI, A.F.; BORGHEZAN, M.; GUERRA, M.P.; SILVA, A.L.; PORRO, D.; STEFANINI, M.; VIEIRA, H.J. Viticultural performance of Italian grapevines in high altitude regions of Santa Catarina State, Brazil. Acta Horticulturae, v.1115, p.203-210, 2016.

MARCON FILHO, J. L.; HIPÓLITO, J.S.; MACEDO, T.A.; KRETZSCHMAR, A.A.; RUFATO, L. Raleio de cachos sobre o potencial enológico da uva 'cabernet franc' em duas safras. Ciencia Rural, v.45, p.2150-2156, 2015. 
MARCON FILHO, J. L. Sistemas de condução na produção de uvas viníferas e composição química e aromática de vinhos em região de altitude de Santa Catarina. 2016. 188p. Tese (Doutorado) - Universidade do Estado de Santa Catarina, Lages, 2016.

MARCON FILHO, J.L.; ALLEBRANDT, R.; WÜRZ, D.A.; BEM, B.P.; MACEDO, T.A.; KRETZSCHMAR, A.A.; RUFATO, L. Cane pruning on chardonnay grapevine in the high-altitude regions of southern Brazil. BIO Web of Conference, v.7, p.1-4, 2016.

PROTAS, J.F.; CAMARGO, U.; MELLO, L.M. A vitivinicultura brasileira: realidadee perspectivas. 2002. Disponível em: <http://www.cnpuv.embrapa.br/publica/artigos/vitivinicultura/> Acesso em: 26 de dezembro de 2016.

PROTAS, J.F.. A produção de vinhos finos: um flash do desafio brasileiro. Pesquisa Agropecuária Catarinense, v. 21, n.1, 2008.

RUFATO, L.; LERIN, S.; ALLEBRANDT, R.; FAGHERAZZI, A.; MARIO, A.E.; BOFF, C.E.; KRETZSCHMAR, A.A. Abscisic acid applications increases color in grapes and juice of 'isabel'. Acta Horticulturae, v.1115, p.217-224, 2016.

SUGAHARA, C. R. A extensão universitária como ação socioeducativa. Revista Conexão UEPG, v.8, n. 2, 2012.

TERRA, S.T.B.; ZÜGE, D.P.P.O. Floricultura: a produção de flores como uma nova alternativa de emprego e renda para a comunidade de Bagé-RS. Revista Conexão UEPG, v.9, n.2, p. 342353, 2013.

WÜRZ, D.A.; MARCON FILHO, J.L.; ALLEBRANDT, R.; DE BEM, B.P.; OUTEMANE, M.; KRETZSCHMAR, A.A.; RUFATO, L. Diagnóstico do enoturismo na região dos vinhos de altitude de Santa Catarina. Revista Brasileira de Viticultura e Enologia, v.8, p.132-138, 2016. 